\title{
Diverse Consequences of Algorithmic Probability
}

\author{
Eray Özkural \\ Computer Engineering Department, Bilkent University, Ankara, Turkey
}

\begin{abstract}
We reminisce and discuss applications of algorithmic probability to a wide range of problems in artificial intelligence, philosophy and technological society. We propose that Solomonoff has effectively axiomatized the field of artificial intelligence, therefore establishing it as a rigorous scientific discipline. We also relate to our own work in incremental machine learning and philosophy of complexity.
\end{abstract}

\section{Introduction}

Ray Solomonoff was a pioneer in mathematical Artificial Intelligence (AI), whose proposal of Algorithmic Probability (ALP) has led to diverse theoretical consequences and applications, most notably in AI. In this paper, we try to give a sense of the significance of his theoretical contributions, reviewing the essence of his proposal in an accessible way, and recounting a few, seemingly unrelated, diverse consequences which, in our opinion, hint towards a philosophically clear world-view that has rarely been acknowledged by the greater scientific community. That is to say, we try to give the reader a glimpse of what it is like to consider the consequences of ALP, and what ideas might lie behind the theoretical model, as we imagine them.

Let $M$ be a reference machine which corresponds to a universal computer 1 with a prefix-free code. In a prefix-free code, no code is a prefix of another. This is also called a self-delimiting code, as most reasonable computer programming languages are. Solomonoff inquired the probability that an output string $x$ is generated by $M$ considering the whole space of possible programs. By giving each program bitstring $p$ an a priori probability of $2^{-|p|}$, we can ensure that the space of programs meets the probability axioms (by the extended Kraft inequality [2]). In other words, we imagine that we toss a fair coin to generate each bit of a random program. This probability model of programs entails the following probability mass function (p.m.f.) for strings $x \in\{0,1\}^{*}$ :

$$
P_{M}(x)=\sum_{M(p)=x *} 2^{-|p|}
$$

which is the probability that a random program will output a prefix of $x . P_{M}(x)$ is called the algorithmic probability of $x$ for it assumes the definition of program based probability. We use $P$ when $M$ is clear from the context to avoid clutter.

\footnotetext{
${ }^{1}$ Optionally, it can be probabilistic to deal with general induction problems, i.e., it has access to a random number generator [1 Section 4].
} 


\section{Solomonoff Induction}

Using this probability model of bitstrings, one can make predictions. Intuitively, we can state that it is impossible to imagine intelligence in the absence of any prediction ability: purely random behavior is decisively non-intelligent. Since, $P$ is a universal probability model, it can be used as the basis of universal prediction, and thus intelligence. Perhaps, Solomonoff's most significant contributions were in the field of AI, as he envisioned a machine that can learn anything from scratch. Reviewing his early papers such as 34, we see that he has established the theoretical justification for machine learning and data mining fields. Few researchers could ably make claims about universal intelligence as he did. Unfortunately, not all of his ideas have reached fruition in practice; yet there is little doubt that his approach was the correct basis for a science of intelligence.

His main proposal for machine learning is inductive inference [5]6] circa 1964, for a variety of problems such as sequence prediction, set induction, operator induction and grammar induction [7. Without much loss of generality, we can discuss sequence prediction on bitstrings. Assume that there is a computable p.m.f. of bitstrings $P_{1}$. Given a bitstring $x$ drawn from $P_{1}$, we can define the conditional probability of the next bit simply by normalizing (11) [7]. Algorithmically, we would have to approximate (1) by finding short programs that generate $x$ (the shortest of which is the most probable). In more general induction, we run all models in parallel, quantifying fit-to-data, weighed by the algorithmic probability of the model, to find the best models and construct distributions [7]; the common point being determining good models with high a priori probability. Finding the shortest program in general is undecidable, however, Levin search [8] can be used for this purpose. There are two important results about Solomonoff induction that we shall mention here. First, Solomonoff induction converges very rapidly to the real probability distribution. The convergence theorem shows that the expected total square error is related only to the algorithmic complexity of $P_{1}$, which is independent from $x$. The following bound [9] is discussed at length in [10] with a concise proof:

$$
\left.E_{P}\left[\sum_{m=1}^{n}\left(P\left(a_{m+1}=1 \mid a_{1} a_{2} \ldots a_{m}\right)-P_{1}\left(a_{m+1}=1 \mid a_{1} a_{2} \ldots a_{m}\right)\right)^{2}\right] \leq-\frac{1}{2} \ln P\left(P_{1}\right)\right)
$$

This bound characterizes the divergence of the ALP solution from the real probability distribution $P_{1} . P\left(P_{1}\right)$ is the a priori probability of $P_{1}$ p.m.f. according to our universal distribution $P_{M}$. On the right hand side of (2), $-\ln P_{M}\left(P_{1}\right)$ is roughly $k \ln 2$ where $k$ is the Kolmogorov complexity of $P_{1}$ (the length of the shortest program that defines it), thus the total expected error is bounded by a constant, which guarantees that the error decreases very rapidly as example size increases. Secondly, there is an optimal search algorithm to approximate Solomonoff induction, which adopts Levin's universal search method to solve the problem of universal induction 811. Universal search procedure time-shares all candidate programs according to their a priori probability with a clever watchdog policy to avoid the practical impact of the undecidability of the halting 
problem 11. The search procedure starts with a time limit $t=t_{0}$, in its iteration tries all candidate programs $c$ with a time limit of $t . P(c)$, and while a solution is not found, it doubles the time limit $t$. The time $t(s) / P(s)$ for a solution program $s$ taking time $t(s)$ is called the Conceptual Jump Size (CJS), and it is easily shown that Levin Search terminates in at most 2.CJS time. To obtain alternative solutions, one may keep running after the first solution is found, as there may be more probable solutions that need more time. The optimal solution is computable only in the limit, which turns out to be a desirable property of Solomonoff induction, as it is complete and uncomputable [12, Section 2]. An explanation of Levin's universal search procedure and its application to Solomonoff induction may be found in 81113.

\section{The Axiomatization of Artificial Intelligence}

We believe in fact that Solomonoff's work was seminal in that he has singlehandedly axiomatized AI, discovering the minimal necessary conditions for any machine to attain general intelligence (based on our interpretation of [1]).

Informally, these axioms are:

AI0. AI must have in its possession a universal computer M (Universality).

AI1. AI must be able to learn any solution expressed in M's code (Learning recursive solutions).

AI2. AI must use probabilistic prediction (Bayes' theorem).

AI3. AI must embody in its learning a principle of induction (Occam's razor).

While it may be possible to give a more compact characterization, these are ultimately what is necessary for the kind of general learning that Solomonoff induction achieves. ALP can be seen as a complete formalization of Occam's razor (as well as Epicurus's principle) [14] and thus serve as the foundation of universal induction, capable of solving all AI problems of significance. The axioms are important because they allow us to assess whether a system is capable of general intelligence or not.

Obviously, AI1 entails AI0, therefore AI0 is redundant, and can be omitted entirely, however we stated it separately only for historical reasons, as one of the landmarks of early AI research, in retrospect, was the invention of the universal computer, which goes back to Leibniz's idea of a universal language (characteristica universalis) that can express every statement in science and mathematics, and has found its perfect embodiment in Turing's research [1516]. A related achievement of early AI was the development of LISP, a universal computer based on lambda calculus (which is a functional model of computation) that has shaped much of early AI research.

See also a recent survey about inductive inference [17] with a focus on Minimum Message Length (MML) principle introduced in 1968 [18. MML principle is also a formalization of induction developed within the framework of classical information theory, which establishes a trade-off between model complexity and fit-to-data by finding the minimal message that encodes both the model 
and the data [19. This trade-off is quite similar to the earlier forms of induction that Solomonoff developed, however independently discovered. Dowe points out that Occam's razor means choosing the simplest single theory when data is equally matched, which MML formalizes perfectly (and is functional otherwise in the case of inequal fits) while Solomonoff induction maintains a mixture of alternative solutions [17, Sections $2.4 \& 4$ ]. On the other hand, the diversity of solutions in ALP is seen as desirable by Solomonoff himself [12, and in a recent philosophical paper which illustrates how Solomonoff induction dissolves various philosophical objections to induction [14]. Nevertheless, it is well worth mentioning that Solomonoff induction (formal theory published in 1964 [56]), MML (1968), and Minimum Description Length 20 formalizations, as well as Statistical Learning Theory [21] (initially developed in 1960), all provide a principle of induction (AI3). However, it was Solomonoff who first observed the importance of universality for AI (AI0-AI1). The plurality of probabilistic approaches to induction supports the importance of AI3 (as well as hinting that diversity of solutions may be useful). AI2, however, does not require much explanation. Some objections to Bayesianism are answered using MML in 22. Please also see an intruging paper by Wallace and Dowe [23] on the relation between MML and Kolmogorov complexity, which states that Solomonoff induction is tailored to prediction rather than inference, and recommends non-universal models in practical work, therefore becomes incompatible with the AI axioms (AI0-AI1). Ultimately, empirical work will illuminate whether our AI axioms should be adopted, or more restrictive models are sufficient for universal intelligence; therefore such alternative viewpoints must be considered. In addition to this, Dowe discusses the relation between inductive inference and intelligence, and the requirements of intelligence as we do elsewhere [17, Section 7.3]. Also relevant is an adaptive universal intelligence test that aims to measure the intelligence of any AI agent, and discusses various definitions of intelligence [24].

\section{Incremental Machine Learning}

In solving a problem of induction, the aforementioned search methods suffer from the huge computational complexity of trying to compress the entire input. For instance, if the complexity of the p.m.f. $P_{1}$ is about 400 bits, Levin search would take on the order of $2^{400}$ times the running time of the solution program, which is infeasible (quite impossible in the observed universe). Therefore, Solomonoff has suggested using an incremental machine learning algorithm, which can re-use information found in previous solutions [13].

The following argument illustrates the situation more clearly. Let $P_{1}$ and $P_{2}$ be the p.m.f.'s corresponding to a training sequence of two induction problems (any of them, not necessarily sequence prediction, to which others can be reduced easily) with data $\left\langle d_{1}, d_{2}>\right.$. Assume that the first problem has been solved (correctly) with universal search. It has taken at most 2. CJS $_{1}=2 . t\left(s_{1}\right) / P\left(s_{1}\right)$ time. If the second problem is solved in an incremental fashion, making use of the information from $P_{1}$, then the running time of discovering a solution $s_{2}$ for 
$d_{2}$ reduces, depending on the success of information transfer across problems. Here, we quantify how much in familiar probabilistic terms.

In 10], Solomonoff describes an information theoretic interpretation of ALP, which suggests the following entropy function:

$$
H^{*}(x)=-\log _{2} P(x)
$$

This entropy function has perfect sub-additivity of information according to the corresponding conditional entropy definition:

$$
\begin{aligned}
P(y \mid x) & =\frac{P(x, y)}{P(x)} \\
H^{*}(y \mid x) & =-\log _{2} P(y \mid x) \\
H^{*}(x, y) & =H^{*}(x)+H^{*}(y \mid x)
\end{aligned}
$$

This definition of entropy thus does not suffer from the additive constant terms as in Chaitin's version. We can instantly define mutual entropy:

$$
H^{*}(x: y)=H^{*}(x)+H^{*}(y)-H^{*}(x, y)=H^{*}(y)-H^{*}(y \mid x)
$$

which trivially follows.

A KUSP machine is a universal computer that can store data and methods in additional storage. In 1984, Solomonoff observed that KUSP machines are especially suitable for incremental learning [11. In our work [25] we found that, the incremental learning approach was indeed useful (as in the preceding OOPS algorithm [26]). Here is how we interpreted incremental learning. After each induction problem, the p.m.f. $P$ is updated, thus for every new problem a new probability distribution is obtained. Although we are using the same $M$ reference machine for trial programs, we are referring to implicit KUSP machines which store information about the experience of the machine so far, in subsequent problems. In our example of two induction problems, let the updated $P$ be called $P^{\prime}$, naturally there will be an update procedure which takes time $t_{u}\left(P, s_{1}\right)$. Just how much time can we expect to save if we use incremental learning instead of independent learning? First, let us write the time bound 2.t $(s) / P(s)$ as $t(s) .2^{H^{*}(s)+1}$. If $s_{1}$ and $s_{2}$ are not algorithmically independent, then $H^{*}\left(s_{2} \mid s_{1}\right)$ is smaller than $H^{*}\left(s_{2}\right)$. Independently, we would have $t\left(s_{1}\right) \cdot 2^{H^{*}\left(s_{1}\right)+1}+t\left(s_{2}\right) \cdot 2^{H^{*}\left(s_{2}\right)+1}$, together, we will have, in the best case $t\left(s_{1}\right) \cdot 2^{H^{*}\left(s_{1}\right)+1}+t\left(s_{2}\right) \cdot 2^{H^{*}\left(s_{2} \mid s_{1}\right)+1}$ for the search time, assuming that recalling $s_{1}$ takes no time for the latter search task (which is an unrealistic assumption). Therefore in total, the latter search task can accelerate $2^{H^{*}(s 1: s 2)}$ times, and we can save $t\left(s_{2}\right) \cdot 2^{H^{*}\left(s_{2}\right)+1}\left(1-2^{-H^{*}(s 1: s 2)}\right)-t_{u}\left(P, s_{1}\right)$ total time in the best case (only an upper bound since we did not account for recall time). Note that the maximum temporal gain is related to both how much mutual information is discovered across solutions (thus $P_{i}$ 's), and how much time the update procedure takes. Clearly, if the update time dominates overall, incremental learning is in vain. However, if updates are effective and efficient, there is enormous potential in incremental machine learning. 
During the experimental tests of our Stochastic Context Free Grammar based search and update algorithms [25], we have observed that in practice we can realize fast updates, and we can still achieve actual code re-use and tremendous speed-up. Using only 0.5 teraflop/sec of computing speed and a reference machine choice of R5RS Scheme [27, we solved 6 simple deterministic operator induction problems in 245.1 seconds. This running time is compared to 7150 seconds without any updates. Scaled to human-level processing speed of 100 teraflop/sec, our system would learn and solve the entire training sequence in 1.25 seconds, which is (arguably) better than most human students. In one particular operator induction problem (fourth power, $x^{4}$ ), we saw actual code re-use: (define (pow4 $\mathrm{x})$ (define $(\operatorname{sqr} \mathrm{x})(* \mathrm{x} \mathrm{x})$ ) (sqr $(\operatorname{sqr} \mathrm{x})$ )), and an actual speedup of 272 . The gains that we saw confirmed the incremental learning proposals of Solomonoff, mentioned in a good number of his publications, but most clearly in 11131. Based on our work and the huge speedup observed in OOPS for a shorter training sequence [26], we have come to believe that incremental learning has the epistemological status of an additional AI axiom:

AI4. AI must be able to use its previous experience to speed up subsequent prediction tasks (Transfer Learning).

This axiom is justified by observing that many universal induction problems are completely unsolvable by a system that does not have the adequate sort of algorithmic memory, regardless of the search method.

The results above may be contrasted with inductive programming approaches, since we predicted deterministic functions. One of the earliest and most successful inductive programming systems is ADATE, which is optimized for a more specific purpose. ADATE system has yielded impressive results in an ML variant by user supplied primitives and constraining candidate programs [28]. Universal representations have been investigated in inductive logic programming as well [29], however U-learning unfortunately lacks the extremely accurate generalization of Solomonoff induction. It has been shown that incremental learning is useful in the inductive programming framework [30], which supports our observation of the necessity of incremental machine learning. Another relevant work is a typed higher-order logic knowledge representation scheme based on term representation of individuals and a rich representation language encompassing many abstract data types [31. A recent survey on inductive programming may be found in 32 .

We should also account our brief correspondence with Solomonoff. We expressed that the prediction algorithms were powerful but it seemed that memory was not used sufficiently. Solomonoff responded by mentioning the potential stochastic grammar and genetic programming approaches that he was working on at the time. Our present research was motivated by a problem he posed during the discussions of his seminars in Turing Days '06 at Bilgi University, Istanbul: "We can use grammar induction for updating a stochastic context free grammar, but there is a problem. We already know the grammar of the reference machine.". We designed our incremental learning algorithms to address this 
particular problem2. Solomonoff has also guided our research by making a valuable suggestion, that it is more important to show whether incremental learning works over a sequence of simpler problems than solving a difficult problem. We have in addition investigated the use of PPM family of compressors following his proposal, but as we expected, they were not sufficient for guiding LISP-like programs, and would require too many changes. Therefore, we proceeded directly to the simplest kind of guiding p.m.f. that would work for Scheme, as we preferred not to work on assembly-like languages for which PPM might be appropriate, since, in our opinion, high-level languages embody more technological progress (see also [33] which employs a Scheme subset). Colorfully speaking, inventing a functional form in assembly might be like re-inventing the wheel. However, in general, it would not be trivial for the induction system to invent syntax forms that compare favorably to LISP, especially during preliminary training. Therefore, much intelligence is already present in a high-level universal computer (AI0) which we simply take advantage of.

\section{Cognitive Architecture}

Another important discussion is whether a cognitive architecture is necessary. The axiomatic approach was seen counter-productive by some leading researchers in the past. However, we think that their opinion can be expressed as follows: the minimal program that realizes these axioms is not automatically intelligent, because in practice an intelligent system requires a good deal of algorithmic information to take off the ground. This is not a bad argument, since obviously, the human brain is well equipped genetically. However, we cannot either rule out that a somewhat compact system may achieve human-level general intelligence. The question therefore, is whether a simply described system like AIXI [34] (an extension of Solomonoff induction to reinforcement learning) is sufficient in practice, or there is a need for a modular/extensible cognitive architecture that has been designed in particular ways to promote certain kinds of mental growth and operation. Some proponents of general purpose AI research think that such a cognitive architecture is necessary, e.g., OpenCog [35. Schmidhuber has suggested the famous Gödel Machine which has a mechanical model of machine consciousness 36. Solomonoff himself has proposed early on in 2002, the design of Alpha, a generic AI architecture which can ultimately solve free-form timelimited optimization problems [13. Although in his later works, Solomonoff has not made much mention of Alpha and has instead focused on the particulars of the required basic induction and learning capability, nonetheless his proposal remains as one of the most extensible and elegant self-improving AI designs.

\footnotetext{
${ }^{2}$ We occassionally corresponded via e-mail. Before the AGI-10 conference, he had reviewed a draft of my paper, and he had commented that the "learning programming idioms" and "frequent subprogram mining" algorithms were interesting, which was all the encouragement I needed. The last e-mail I received from him was on 11/Oct/2009. I regretfully learnt that he passed away a month later. His independent character and true scientific spirit will always be a shining beacon for me.
} 
Therefore, this point is open to debate, though some researchers may want to assume another, entirely optional, axiom:

AI5. AI must be arranged such that self-improvement is feasible in a realistic mode of operation (Cognitive Architecture).

It is doubtful for instance whether a combination of incremental learning and AIXI will result in a practical reinforcement learning agent. Neither is it well understood whether autonomous systems with built-in utility/goal functions are suitable for all practical purposes. We anticipate that such questions will be settled by experimenters, as the complexity of interesting experiments will quickly overtake theoretical analysis.

We do not consider human-like behavior, or a robotic body, or an autonomous AI design, such as a goal-driven or reinforcement-learning agent, essential to intelligence, hence we did not propose autonomy or embodiment as an axiom. Solomonoff has commented likewise on the preferred target applications [37]:

To start, I'd like to define the scope of my interest in A.I. I am not particularly interested in simulating human behavior. I am interested in creating a machine that can work very difficult problems much better and/or faster than humans can - and this machine should be embodied in a technology to which Moore's Law applies. I would like it to give a better understanding of the relation of quantum mechanics to general relativity. I would like it to discover cures for cancer and AIDS. I would like it to find some very good high temperature superconductors. I would not be disappointed if it were unable to pass itself off as a rock star.

\section{Philosophical Foundation and Consequences}

Solomonoff's AI theory is founded on a wealth of philosophy. Here, we shall briefly revisit the philosophical foundation of ALP and point out some of its philosophical consequences. In his posthumous publication, Solomonoff mentions the inspiration for some of his work: Carnap's idea that the state of the world can be represented by a finite bitstring (and that science predicts future bits with inductive inference), Turing's universal computer (AI0) as communicated by Minsky and McCarthy, and Chomsky's generative grammars 12 . The discovery of ALP is described by Solomonoff in quite a bit of detail in [38], which relates his discovery to the background of many prominent thinkers and contributors. Carnap's empiricism seems to have been a highly influential factor in Solomonoff's research as he sought to find how science is carried out, rather than particular scientific findings; and ALP is a satisfactory solution to Carnap's program of inductive inference [14].

Let us then recall some philosophically relevant aspects of ALP discussed in the most recent publications of Solomonoff. First, the exact same method is used to solve both mathematical and scientific problems. This means that there is no fundamental epistemological difference between these problems; our interpretation is that, this is well founded only when we observe that mathematical 
problems themselves are computational or linguistic problems, in practice mathematical problems can be reduced to particular computational problems, and here is why the same method works for both kinds of problems. Mathematical facts do not preside over or precede physical facts, they themselves are solutions of physical problems ultimately (e.g., does this particular kind of machine halt or not?). And the substance of mathematics, the lucid sort of mathematical language and concepts that we have invented, can be fully explained by Solomonoff induction, as those are the kinds of useful programs, which have aided an intellect in its training, and therefore are retained as linguistic and algorithmic information. The subjectivity and diversity aspects of ALP [12, Sections 3 \& 4] fully explain why there can be multiple and almost equally productive foundations of mathematics, as those merely point out somewhat equally useful formalisms invented by different mathematicians. There is absolutely nothing special about ZFC theory, it is just a formal theory to explain some useful procedures that we perform in our heads, i.e., it is more like the logical explanation of a set module in a functional programming language than anything else, however, the operations in a mathematician's brain are not visible to their owner, thereby leading to useless Platonist fantasies of some mathematicians owing to a dearth of philosophical imagination. Therefore, it does not matter much whether one prefers this or that formalization of set theory, or category theory as a foundation, unless that choice restricts success in the solution of future scientific problems. Since, such a problematic scientific situation does not seem to have emerged yet (forcing us to choose among particular formalizations), the diversity principle of ALP forces us to retain them all. That is to say, subscribing to the ALP viewpoint has the unexpected consequence that we abandon both Platonism and Formalism. There is a meaning in formal language, in the manner which improves future predictions, however, there is not a single a priori fact, in addition to empirical observations, and no such fact is ever needed to conduct empirical work, except a proper realization of axioms A1-A3 (and surely no sane scientist would accept that there is a unique and empty set that exists in a hidden order of reality). When we consider these axioms, we need to understand the universality of computation, and the principled manner in which we have to employ it for reliable induction in our scientific inquiries. The only physically relevant assumption is that of the computability of the distributions which generate our empirical problems (regardless of whether the problem is mathematical or scientific), and the choice of a universal computer which introduces a necessary subjectivity. The computability aspect may be interpreted as information finitism, all the problems that we can work with should have finite entropy. Yet, this restriction on disorder is not at all limiting, for it is hardly conceivable how one may wish to solve a problem of actually infinite complexity. Therefore, this is not much of an assumption for scientific inquiry, especially given that both quantum mechanics and general relativity can be described in computable mathematics (see for instance [39] about the applicability of computable mathematics to quantum mechanics). And neither can one hope to find 
an example of a single scientifically valid problem in any textbook of science that requires the existence of distributions with infinite complexity to solve.

With regards to general epistemology, ALP/AIT may be seen as largely incompatible with non-reductionism. Non-reductionism is quite misleading in the manner it is usually conveyed. Instead, we must seek to understand irreducibility in the sense of AIT, of quantifying algorithmic information, which allows us to reconcile the concept of irreducibility with physicalism (which we think every empiricist should accept) [40]. In particular, we can partially formalize the notion of knowledge by mutual information between the world and a brain. Our paper proposed a physical solution to the problem of determining the most "objective" universal computer: it is the universe itself. If digital physics were true, this might be for instance a particular kind of graph automata, or if quantum mechanics were the basis, then a universal quantum computer could be used; however, for many tasks using such a low-level computer might be extraordinarily difficult. We also argued that extreme non-reductionism leads to arguments from ignorance such as ontological dualism, and information theory is much better suited to explaining evolution and the need for abstractions in our language. It should also be obvious that the ALP solution to AI extends the two main tenets of logical positivism, which are verificationism and unified science, as it gives a finite cognitive procedure with which one can conduct all empirical work, and allows us to develop a private language with which we can describe all of science and mathematics. However, we should also mention that this strengthened positivism does not require a strict analytic-synthetic distinction; a spectrum of analytic-synthetic distinction as in Quine's philosophy seems to be acceptable [41. We have already seen that according to ALP, mathematical and scientific problems have no real distinction, therefore like Quine, ALP would allow revising even mathematical logic itself, and we need not remind that the concept of universal computer itself has not appeared out of thin air, but has been invented due to the laborious mental work of scientists, as they abstracted from the mechanics of performing mathematics; at the bottom these are all empirical problems [42. On the other hand, a "web of belief" as in Quine, by no means suggests non-reductionism, for that could be true only if indeed there were phenomena that had unscathable (infinite) complexity, such as Turing oracle machines which were not proposed as physical machines, but only as a hypothetical concept [16. Quine himself was a physicalist; we do not think that he would support the later vendetta against reductionism which may be a misunderstanding of his holism. Though, it may be argued that his obscure version of Platonism, which does not seem much scientific to us, may be the culprit. Today's Bayesian networks seem to be a good formalization of Quine's web of belief, and his instrumentalism is consistent with the ALP approach of maintaining useful programs. Therefore, on this account, psychology ought to be reducible to neurophysiology, as the concept of life to molecular biology, because these are all ultimately sets of problems that overlap in the physical world, and the relation between them cannot hold an infinite amount of information; which would require an infinitely complex local environment, and that does not seem 
consistent with our scientific observations. That is to say, discovery of bridge disciplines is possible as exemplified by quantum chemistry and molecular biology, and it is not different from any other kind of empirical work. Recently, it has been perhaps better understood in the popular culture that creationism and non-reductionism are almost synonymous (regarding the claims of "intelligent design" that the flagella of bacteria are too complex to have evolved). Note that ALP has no qualms with the statistical behavior of quantum systems, as it allows non-determinism. Moreover, the particular kind of irreducibility in AIT corresponds to weak emergentism, and most certainly contradicts with strong emergentism which implies supernatural events. Please see also [17, Section 7] for a discussion of philosophical problems related to algorithmic complexity.

\section{Intellectual Property towards Infinity Point}

Solomonoff has proposed the infinity point hypothesis, also known as the singularity, as an exponentially accelerating technological progress caused by humanlevel AI's that complement the scientific community, to accelerate our progress ad infinitum within a finite, short time (in practice only a finite, but significant factor of improvement could be expected) in 1985 [43] (the first paper on the subject). Solomonoff has proposed seven milestones of AI development: A: modern AI phase (1956 Dartmouth conference), B: general theory of problem solving (our interpretation: Solomonoff Induction, Levin Search), C: self-improving AI (our interpretation: Alpha architecture, 2002), D: AI that can understand English (our interpretation: not realized yet), E: human-level AI, F: an AI at the level of entire computer science (CS) community, G: an AI many times smarter than the entire CS community.

A weak condition for the infinity point may be obtained by an economic argument, also covered in [43] briefly. The human brain produces 5 teraflops/watt roughly. The current incarnation of NVIDIA's General Purpose Graphics Programming Unit architectures called Fermi achieves about 6 gigaflops/watt [44]. Assuming 85\% improvement in power efficiency per year (as seen in NVIDIA's projections), in 12 years, human-level energy efficiency of computing will be achieved. After that date, even if mathematical AI fails due to an unforeseen problem, we will be able to run our brain simulations faster than us, using less energy than humans, effectively creating a bio-information based AI which meets the basic requirement of infinity point. For this to occur, whole brain simulation projects must be comprehensive in operation and efficient enough 45. Otherwise, human-level AI's that we will construct should match the computational efficiency of the human brain. This weaker condition rests on an economic observation: the economic incentive of cheaper intellectual work will drive the proliferation of personal use of brain simulations. According to NVIDIA's projections, thus, we can expect the necessary conditions for the infinity point to materialize by 2023, after which point technological progress may accelerate very rapidly. According to a recent paper by Koomey, the energy efficiency of computing is doubling every 1.5 years (about $60 \%$ per year), regardless of architecture, which would set the date at 2026 [46]. 
Assume that we are progressing towards the hypothetical infinity point. Then, the entire human civilization may be viewed as a global intelligence working on technological problems. The practical necessity of incremental learning suggests that when faced with more difficult problems, better information sharing is required. If no information sharing is present between researchers (i.e., different search programs), then, they will lose time traversing overlapping program subspaces. This is most clearly seen in the case of simultaneous inventions when an idea is said to be "up in the air" and is invented by multiple, independent parties on near dates. If intellectual property (IP) laws are too rigid and costly, this would entail that there is minimal information sharing, and after some point, the global efficiency of solving non-trivial technological problems would be severely hampered. Therefore, to utilize the infinity point effects better, knowledge sharing must be encouraged in the society. Maximum efficiency in this fashion can be provided by free software licenses, and a reform of the patent system. Our view is that no single company or organization can (or should) have a monopoly on the knowledge resources to attack problems with truly large algorithmic complexity (monopoly is mostly illegal presently at any rate). We tend to think that sharing science and technology is the most efficient path towards the infinity point. Naturally, free software philosophy is not acceptable to much commercial enterprise, thus we suggest that as technology advances, the overhead of enforcing IP laws are taken into account. If technology starts to advance much more rapidly, the duration of the IP protection may be shortened, for instance, as after the AI milestone F, the bureaucracy and restrictions of IP law may be a serious bottleneck.

\section{Conclusion}

We have mentioned diverse consequences of ALP in axiomatization of AI, philosophy, and technological society. We have also related our own research to Solomonoff's proposals. We interpret ALP and AIT as a fundamentally new world-view which allows us to bridge the gap between complex natural phenomena and positive sciences more closely than ever. This paradigm shift has resulted in various breakthrough applications and is likely to benefit the society in the foreseeable future.

Acknowledgements. We thank anonymous reviewers, David Dowe and Laurent Orseau for their valuable comments, which substantially improved this paper.

\section{References}

1. Solomonoff, R.J.: Progress in incremental machine learning. Technical Report IDSIA-16-03, IDSIA, Lugano, Switzerland (2003)

2. Chaitin, G.J.: A theory of program size formally identical to information theory. J. ACM 22, 329-340 (1975) 
3. Solomonoff, R.J.: An inductive inference machine. Dartmouth Summer Research Project on Artificial Intelligence (1956) A Privately Circulated Report

4. Solomonoff, R.J.: An inductive inference machine. In: IRE National Convention Record, Section on Information Theory, Part 2, New York, USA, pp. 56-62 (1957)

5. Solomonoff, R.J.: A formal theory of inductive inference, part i. Information and Control 7(1), 1-22 (1964)

6. Solomonoff, R.J.: A formal theory of inductive inference, part ii. Information and Control 7(2), 224-254 (1964)

7. Solomonoff, R.J.: Three kinds of probabilistic induction: Universal distributions and convergence theorems. The Computer Journal 51(5), 566-570 (2008); Christopher Stewart Wallace, memorial special issue (1933-2004)

8. Levin, L.A.: Universal sequential search problems. Problems of Information Transmission 9(3), 265-266 (1973)

9. Solomonoff, R.J.: Algorithmic probability: Theory and applications. In: Dehmer, M., Emmert-Streib, F. (eds.) Information Theory and Statistical Learning, pp. 1-23. Springer Science+Business Media, N.Y. (2009)

10. Solomonoff, R.J.: Complexity-based induction systems: Comparisons and convergence theorems. IEEE Trans. on Information Theory IT-24(4), 422-432 (1978)

11. Solomonoff, R.J.: Optimum sequential search. Technical report, Oxbridge Research, Cambridge, Mass., USA (1984)

12. Solomonoff, R.J.: Algorithmic Probability - Its Discovery - Its Properties and Application to Strong AI. In: Randomness Through Computation: Some Answers, More Questions, pp. 149-157. World Scientific Publishing Company (2011)

13. Solomonoff, R.J.: A system for incremental learning based on algorithmic probability. In: Proceedings of the Sixth Israeli Conference on Artificial Intelligence, Tel Aviv, Israel, pp. 515-527 (1989)

14. Rathmanner, S., Hutter, M.: A philosophical treatise of universal induction. Entropy 13(6), 1076-1136 (2011)

15. Davis, M.: The Universal Computer: The Road from Leibniz to Turing. W. W. Norton \& Company (2000)

16. Turing, A.M.: On computable numbers, with an application to the entscheidungsproblem. Proceedings of the London Mathematical Society s2-42(1), 230-265 (1937)

17. Dowe, D.L.: MML, hybrid Bayesian network graphical models, statistical consistency, invariance and uniqueness. In: Handbook of the Philosophy of Science (HPS ). Philosophy of Statistics, vol. 7, pp. 901-982. Elsevier (2011)

18. Wallace, C.S., Boulton, D.M.: A information measure for classification. Computer Journal 11(2), 185-194 (1968)

19. Wallace, C.S.: Statistical and Inductive Inference by Minimum Message Length. Springer, Berlin (2005)

20. Barron, A., Rissanen, J., Yu, B.: The minimum description length principle in coding and modeling (invited paper), pp. 699-716. IEEE Press, Piscataway (2000)

21. Vapnik, V.: Statistical Learning Theory. John Wiley and Sons, NY (1998)

22. Dowe, D.L., Gardner, S., Oppy, G.: Bayes not bust! why simplicity is no problem for bayesians. The British Journal for the Philosophy of Science 58(4), 709-754 (2007)

23. Wallace, C.S., Dowe, D.L.: Minimum message length and kolmogorov complexity. The Computer Journal 42(4), 270-283 (1999)

24. Hernndez-Orallo, J., Dowe, D.L.: Measuring universal intelligence: Towards an anytime intelligence test. Artificial Intelligence 174(18), 1508-1539 (2010) 
25. Özkural, E.: Towards heuristic algorithmic memory. In: Schmidhuber, J., Thórisson, K.R., Looks, M. (eds.) AGI 2011. LNCS, vol. 6830, pp. 382-387. Springer, Heidelberg (2011)

26. Schmidhuber, J.: Optimal ordered problem solver. Machine Learning 54, 211-256 (2004)

27. Kelsey, R., Clinger, W., Rees, J.: Revised5 report on the algorithmic language scheme. Higher-Order and Symbolic Computation 11(1) (1998)

28. Olsson, J.R.: Inductive functional programming using incremental program transformation. Artificial Intelligence 74, 55-83 (1995)

29. Muggleton, S., Page, C.: A learnability model for universal representations. In: Proceedings of the 4th International Workshop on Inductive Logic Programming, vol. 237, pp. 139-160. Citeseer (1994)

30. Ferri-Ramírez, C., Hernández-Orallo, J., Ramírez-Quintana, M.J.: Incremental learning of functional logic programs. In: Kuchen, H., Ueda, K. (eds.) FLOPS 2001. LNCS, vol. 2024, pp. 233-247. Springer, Heidelberg (2001)

31. Bowers, A., Giraud-Carrier, C., Lloyd, J., Sa, E.: A knowledge representation framework for inductive learning (2001)

32. Kitzelmann, E.: Inductive programming: A survey of program synthesis techniques. In: Schmid, U., Kitzelmann, E., Plasmeijer, R. (eds.) AAIP 2009. LNCS, vol. 5812, pp. 50-73. Springer, Heidelberg (2010)

33. Looks, M.: Scalable estimation-of-distribution program evolution. In: Proceedings of the 9th Annual Conference on Genetic and Evolutionary Computation (2007)

34. Hutter, M.: Universal algorithmic intelligence: A mathematical top $\rightarrow$ down approach. In: Goertzel, B., Pennachin, C. (eds.) Artificial General Intelligence. Cognitive Technologies, pp. 227-290. Springer, Heidelberg (2007)

35. Goertzel, B.: Opencogprime: A cognitive synergy based architecture for artificial general intelligence. In: Baciu, G., Wang, Y., Yao, Y., Kinsner, W., Chan, K., Zadeh, L.A. (eds.) IEEE ICCI, pp. 60-68. IEEE Computer Society (2009)

36. Schmidhuber, J.: Ultimate cognition à la Gödel. Cognitive Computation 1(2), 177-193 (2009)

37. Solomonoff, R.J.: Machine learning - past and future. In: The Dartmouth Artificial Intelligence Conference, pp. 13-15 (2006)

38. Solomonoff, R.J.: The discovery of algorithmic probability. Journal of Computer and System Sciences 55(1), 73-88 (1997)

39. Bridges, D., Svozil, K.: Constructive mathematics and quantum physics. International Journal of Theoretical Physics 39, 503-515 (2000)

40. Özkural, E.: A compromise between reductionism and non-reductionism. In: Worldviews, Science and Us: Philosophy and Complexity. World Scientific Books (2007)

41. Quine, W.: Two dogmas of empiricism. The Philosophical Review 60, 20-43 (1951)

42. Chaitin, G.J.: Two philosophical applications of algorithmic information theory. In: Calude, C.S., Dinneen, M.J., Vajnovszki, V. (eds.) DMTCS 2003. LNCS, vol. 2731, pp. 1-10. Springer, Heidelberg (2003)

43. Solomonoff, R.J.: The time scale of artificial intelligence: Reflections on social effects. Human Systems Management 5, 149-153 (1985)

44. Glaskowsk, P.N.: Nvidia's fermi: The first complete gpu computing architecture (2009)

45. Sandberg, A., Bostrom, N.: Whole brain emulation: A roadmap. Technical report, Future of Humanity Institute, Oxford University (2008)

46. Koomey, J.G., Berard, S., Sanchez, M., Wong, H.: Implications of historical trends in the electrical efficiency of computing. IEEE Annals of the History of Computing 33, 46-54 (2011) 